\title{
Pure jump Lévy processes for asset price modelling
}

\author{
Hélyette Geman *
}

ESSEC, Finance Department and University Paris Dauphine, Place de Lattre de Tassigny, 75775 Paris Cedex 16, France

\begin{abstract}
The goal of the paper is to show that some types of Lévy processes such as the hyperbolic motion and the CGMY are particularly suitable for asset price modelling and option pricing. We wish to review some fundamental mathematic properties of Lévy distributions, such as the one of infinite divisibility, and how they translate observed features of asset price returns. We explain how these processes are related to Brownian motion, the central process in finance, through stochastic time changes which can in turn be interpreted as a measure of the economic activity. Lastly, we focus on two particular classes of pure jump Lévy processes, the generalized hyperbolic model and the CGMY models, and report on the goodness of fit obtained both on stock prices and option prices.
\end{abstract}

(C) 2002 Published by Elsevier Science B.V.

JEL classification: G12; G13

Keywords: Stochastic time changes; Economic time; Lévy density; Quadratic variation; Option pricing

\section{Introduction}

Normality of asset returns has played a central role in financial theory for the last few decades, starting with the Markowitz frontier and the Capital Asset Pricing Model and more recently, as a convenient setting for Value at Risk computations. The normality of distributions has been augmented with the assumption of continuity of trajectories when Samuelson introduced in 1965 the geometric Brownian

\footnotetext{
${ }^{*}$ Fax: +33-1-34433001.

E-mail address: geman@essec.fr (H. Geman).
} 
motion, then used in the seminal papers by Black-Scholes (1973) and Merton (1973). As documented in a considerable number of papers written by academics and practitioners, both normality and continuity assumptions (which are not identical but related in a way that we shall make precise later on in the paper) are contradicted by the data in several pieces of evidence.

Return distributions are more leptokurtic than the normal one as noted by Fama as early as 1963; this feature is more accentuated when the holding period becomes shorter and becomes particularly clear on high frequency data. Option prices exhibit the famous volatility smile as well as prices higher than predicted by the BlackScholes formula for short-dated options. At the same time, jumps may clearly be identified in equity data; in fact, the inability to trade continuously implies de facto jumps in prices. These jumps contribute to (or may be the source of) stochastic volatility while they lead to finite variation trajectories in the absence of a diffusion term, as observed in practice.

The goal of the paper is to go beyond the Brownian-motion compound Poisson process introduced by Merton in 1976 (and usually referred to as jump-diffusion) and analyze as a better alternative two particular classes of pure jump Lévy processes on which several authors have focused attention lately. These processes are the generalized hyperbolic motion and the CGMY process which have been introduced in the finance literature by Eberlein (1995), Barndorff-Nielsen (1998), on one hand, Geman et al. (2001), Carr et al. (2002) on the other hand.

We also show how that they are related to Brownian motion, a process which does exhibit both properties of normality and continuity mentioned. This relationship holds through a stochastic time change which is in fact a measure of the economic activity as conjectured in Clark's (1973) seminal paper, and demonstrated by Geman and Ané (1996) and Ané and Geman (2000).

The remainder of the paper is organized as follows. Section 2 recalls some fundamental properties of Lévy processes and the financial interpretation attached to them. Section 3 establishes that these processes are necessarily time-changed Brownian motion in a no-arbitrage framework and discusses the properties of the Lévy measure in terms of those of the economic clock. Section 4 describes some pure jump Lévy processes which have been recently demonstrated as providing a conclusive goodness of fit of equity markets. The first class is the class of the generalized hyperbolic motion, which includes the normal inverse Gaussian process and the hyperbolic motion; the second one contains the VG and the CGMY models. Section 5 contains concluding comments.

\section{Some fundamentals of Lévy processes}

As usual in the finance literature, we represent the uncertainty of the economy by a filtered probability space, $\left(\Omega, F, F_{t}, P\right)$ where $F_{t}$ is the filtration of information available at time $t$ and $P$ is the real probability measure. There will be no change of probability measure in this section and all definitions and properties of the processes under analysis will hold with respect to $P$. The Lévy processes, which include Poisson 
process and Brownian motion as special cases, were the first class of stochastic processes to be studied in the spirit of trajectories, crucial for finance. For notational simplicity, we are going to consider $\mathbb{R}$-valued processes starting at zero but most of the results described below hold for $\mathbb{R}^{n}$-valued processes.

A process $X$ is called a Lévy process if it has (almost surely) right-continuous paths and if its increments are independent and time-homogeneous. The first condition is somewhat technical and ensures that the paths of $X$ cannot explode; the second one characterizes Lévy processes from a modelling standpoint and expresses that for any time $t$ greater than $s$, the distribution of the increment $\left(X_{t}-X_{s}\right)$ depends only on the length of the interval $(t-s)$ and that $\left(X_{t}-X_{s}\right)$ is independent of $\left(X_{u}\right.$, $u \leqslant s)$.

An important consequence is the infinite divisibility of distributions that is derived from the above property. For simplicity, let us consider $X_{1}$ (value at the process at time 1): for any $n>1$, we can write

$$
X_{1}=\left(X_{1}-X_{\frac{n-1}{n}}\right)+\left(X_{\frac{n-1}{n}}-X_{\frac{n-2}{n}}\right) t+\left(X_{\frac{2}{n}}-X_{\frac{1}{n}}\right)+X_{\frac{1}{n}} .
$$

Hence $X_{1}$ can be expressed as the sum of $n$ independent identically distributed random variables, with common law the law of $X_{\frac{1}{n}}$, i.e., the law of $X_{1}$ is infinitely divisible (the same holds for all $X_{t}$ ). This is to be compared with the well-cited motivation for modelling stock returns by the Gaussian distribution, namely that this distribution is a limiting distribution of sums of $n$ independent random variables (up to a scaling factor) which may be viewed as representing the effects of various shocks in the economy.

A second major result, which has direct implications for option pricing, is the expression of the characteristic function of $X_{t}$, known as the Lévy-Khintchine formula:

$$
E\left[e^{\mathrm{i} u X_{1}}\right]=\mathrm{e}^{-t \phi(u)}
$$

where $\phi$ has the following Lévy-Khintchine representation:

$$
\phi(u)=\frac{\sigma^{2}}{2} u^{2}-\mathrm{i} a u+\int_{|x| \geqslant 1}\left(1-\mathrm{e}^{\mathrm{i} u x}\right) k(\mathrm{~d} x)+\int_{|x|<1}\left(1-\mathrm{e}^{\mathrm{i} u x}+\mathrm{i} u x\right) k(\mathrm{~d} x) .
$$

$a$ is called the drift of the Lévy process, $\theta$ is the diffusion coefficient and $k(\mathrm{~d} x)$ is a measure on $R-\{0\}$ such that

$$
\int \inf \left(1, x^{2}\right) k(\mathrm{~d} x)<\infty
$$

and called the Lévy measure of the process $X$.

The Lévy measure of the process $X$ may also be defined by

$$
k(A)=E\left\{\sum_{0 \leqslant s \leqslant 1} 1_{A}\left(\Delta X_{s}\right)\right\}
$$

where $A$ is an arbitrary interval bounded away from zero. The Lévy density has the same mathematical requirements as a probability density, except that is does not need to be integrable and must have zero mass at the origin. Integration of the Lévy 
density over a particular spatial domain provides the arrival rates of jump sizes in this domain.

Note that in the whole paper, we shall say that the Lévy process $X$ (which represents the log of the stock price and will be essentiallly a pure jump process) has infinite activity if the integral of the measure $k$ on the real line is infinite. This expression, also used in turbulence theory, characterizes a "high" rate of arrival of jumps of different sizes and will adequately allow us to dispense with the need to consider an additional and unrelated diffusion component. At the other end of the spectrum, the continuity requirement of diffusion models forces the arrival rates of all jumps sizes to zero and thus reduces the local variation of uncertainty in the price dimension to be explained with a single instantaneous volatility parameter.

It is interesting to observe that there is a one-to-one correspondence between Lévy processes and characteristic functions represented as in (1). More precisely, starting with formula (2), we can build three processes $X^{(1)}, X^{(2)}, X^{(3)}$ as follows: Denoting $X_{t}^{(1)}=-a t+\sigma W_{t}$ where $\left(W_{t}\right)$ is a standard $P$-Brownian motion, the characteristic function of $X^{(1)}$ is straightforward and equal to

$$
\phi_{1}(u)=\mathrm{i} a u+\frac{1}{2} \sigma^{2} u^{2} .
$$

Now, consider the process $X_{t}^{(2)}=\sum_{j=1}^{N_{t}} Y_{j}$ where $N$ is a Poisson process whose intensity $\lambda$ is defined by

$$
\lambda=\int_{|x|>1} k(\mathrm{~d} x)
$$

and $Y_{1}, Y_{2}, \ldots, Y_{n} \ldots$ are independent random variables, independent of the process $N$ and with common distribution

$$
1_{|x|>1} k(\mathrm{~d} x) .
$$

We recognize in $X^{(2)}$ a compound Poisson process whose characteristic function is $\mathrm{e}^{-\phi_{2}(u)}$, where

$$
\phi_{2}(u)=-\int\left(\mathrm{e}^{\mathrm{i} u x}-1\right) 1_{|x|>1} k(\mathrm{~d} x)
$$

It may be proved in the same manner that the last term in formula (2) is the characteristic function of a Lévy process $X^{(3)}$ obtained as a limit of compound Poisson processes (different from $X^{(2)}$ ). Hence

$$
X=X^{(1)}+X^{(2)}+X^{(3)}
$$

is the sum of three Lévy processes independent of one another, hence is a Lévy process as well. The same property holds the other way, meaning that any Lévy process can be written as above:

$$
X=X^{(1)}+X^{(2)}+X^{(3)} \text {. }
$$

Let us observe that the decomposition exhibited in (3) illuminates the fact that, in the same way the instantaneous volatility describes the local uncertainty of a diffusion, the Lévy density describes the local uncertainty of a pure jump process. 
Each of $X^{(1)}, X^{(2)}, X^{(3)}$ is a semimartingale, so is $X$. Hence, any Lévy process is a semimartingale. We know that stock prices have to be semimartingales under the real probability measure $P$ and Lévy processes appear as a wide natural class of candidates for stock prices. The above discussion shows that in order to get continuity for the trajectories of the process $X$, the components $X^{(2)}$ and $X^{(3)}$ need to be zero and the process $X$ to be reduced to

$$
X_{t}^{(1)}=-a t+\sigma W_{t} .
$$

Hence, the important property:

The only Lévy process with continuous paths is the Brownian motion (with drift).

From a finance standpoint, this means that if we start with a Lévy process to describe the return (or natural log of stock), we obtain normality together with continuity; expressed differently, it means that it is necessary to introduce discontinuous Lévy processes whenever deviations from normality are clearly exhibited by the data.

We have so far gathered the important properties:

(a) Lévy processes as the representation of stock returns (or price changes) are consistent with the no-arbitrage assumption.

(b) They have the merit of providing infinitely divisible distributions, hence to express price changes as resulting from a great number of shocks in the economy.

(c) If deviations from normality are clearly exhibited by the data, it becomes necessary to introduce discontinuous Lévy processes, possibly with a diffusion component.

(d) In order to obtain a finite quadratic variation process, the diffusion component must be zero and the process be a pure jump Lévy process.

(The quadratic variation of a process $\left(X_{t}\right)$ over a time interval $[a, b]$ is classically defined as the limit of $\sum_{i=1}^{n}\left[X\left(t_{i+1}\right)-X\left(t_{i}\right)\right]^{2}$ when the width of the partition $t_{0}=$ $a<t_{1} \ldots<t_{n+1}=b$ of the interval $[a, b]$ goes to zero.)

Obviously, a finite variation process is a better representation of real stock prices.

\section{Economic activity, time changes and asset price modelling}

In order to provide an answer to the deviations of stock returns from normality already observed at the time, Mandelbrot (1963) proposed the class of stable Pareto distributions, denoted by $\operatorname{SP}(\alpha)$, where $\alpha$, the characteristic exponent, belongs to the interval $] 0,2]$.

In the symmetric case, stable Pareto distributions are defined by the log-characteristic function

$$
\ln \phi(t)=\mathrm{i} \delta t-c|t|^{\alpha}
$$

where $\delta$ denotes the location parameter, $c$ the scale parameter. When the characteristic exponent $\alpha=2$, the stable distribution coincides with the normal distribution; 
for $\alpha=1$, it gives the Cauchy distribution. For $\alpha<2$, stable distributions are more peaked around the center than the normal ones and have arbitrarily fat tails since the variance is infinite. For $\alpha \leqslant 1$, even the first moment does not exist. So far, these features have led most authors to reject the stable hypothesis for stock price returns. Since stable processes are Lévy processes, this shows that some additional properties must be taken into account to identify suitable ones.

In 1973, Clark offers a strictly different solution to account for the non-normality of returns since the title of his paper mentions "a process model with finite variance". Analyzing a database of cotton future price returns, he proposes linking them to the existence of variations in volume during different trading period and introduces subordinated processes by writing the return process $X(t)$ as a subordinated process

$$
X(t)=Z(T(t)) \text {. }
$$

According to the definition given by Bochner in 1955, a subordinator $T(t)$ is an almost surely increasing process with independent and stationary increments; no specific condition is required on the process $Z$ which is sometimes called in finance the directing process.

In his seminal paper, Clark conjectured that $Z$ could be chosen as a Brownian motion, that $T(t)$ be log-normally distributed and have the economic interpretation of (cumulated) volume traded in the market. Indeed, using historical data on returns (represented by $X$ ) and volume (represented by $T$ ), he was able to show that the distribution of $Z$ did satisfy classical normality tests.

Geman and Ané (1996) and Ané and Geman (2000) validate mathematically and extend Clark's conjecture by exhibiting two types of results:

(a) A remarkable theorem by Monroe (1978) establishes that any semimartingale can be written as a time-changed Brownian motion. Since the no-arbitrage assumption implies the existence of a probability measure under which discounted stock prices are martingales, these stock prices have to be semimartingales under the real probability measure. Hence the same property holds for the log of the price and

$$
\ln S(t)=W(T(t))
$$

where we now know that the directing process $W$ is a Brownian motion.

Comparing formula (5) with formula (4), we observe that the left-hand sides are similar since the semimartingale property may be written indifferently for $S$ or $\ln S$. In the right-hand sides however, $T(t)$ is in formula (5) a general time change, only constrained by the property of being an almost surely increasing process.

(b) Given the considerable amount of empirical literature analyzing the relationship between volume, price changes, volatility, information arrival, number of trades (see for instance Karpoff, 1987; Jones et al., 1994), Ané and Geman (2000) test the number of trades against volume as the possible representation of the time change. They conclude through the empirical analysis of high frequency data 
of equity indexes and individual stocks that the (cumulative) number of trades is a better stochastic clock for generating virtually perfect normality of returns.

Formula (5) illuminates how asset prices respond to the arrival of information. Some days, very little news, good or bad, is released; trading is typically slow and prices barely fluctuate. In contrast, when new information arrives and traders adjust their expectations accordingly, trading becomes brisk and the price evolution accelerates.

Before turning to the discussion of the different processes generated for the stock price by interesting choices of the time change $T$, it is worth noticing that the constant volatility in the arithmetic Brownian $W$ is going to give rise to stochastic volatility for the stock price process $S$ when it is "compounded" with a stochastic time $T$. Geman and Yor (1993) who introduced general time changes for solving the valuation of an Asian option in the classical Black-Scholes setting also observe how these time changes appear as a natural tool to handle stochastic volatility. Taking the Hull and White (1987) model where the squared volatility is supposed to be driven by a geometric Brownian motion, they use a stochastic time change to solve for the average "perfect replication time" of put options in portfolio insurance strategies. Following the Geman-Yor representation of time-varying volatility through time change, itself in turn expressed by the economic clock, Ané and Geman (1999) propose an activity-based volatility estimator and show that it performs better than both historical and implied volatilities as a predictor of future realized volatility.

Coming back to formula (5), we observe that two types of randomness come into play in the return process: The Brownian motion and the time change. Due to the self-similarity property of Brownian motion,

$$
W\left(c^{2} t\right)=c W(t) \text { for } c>0,
$$

the qualitative features of trajectories do not change if we change the time-scale. In contrast, real stock price paths change dramatically if we look at them on different time scales (see for instance Table 1).

Table 1

Comparison of skewness and kurtosis at different time scales

\begin{tabular}{llcc}
\hline Mean & Variance & Skewness & Kurtosis \\
\hline $\begin{array}{l}\text { Cisco systems 1-min returns } \\
4.2292 \text { E-06 }\end{array}$ & $4.9975 \mathrm{E}-07$ & $2.2655 \mathrm{E}-01$ & 40.852 \\
$\begin{array}{l}\text { Cisco systems 10-min returns } \\
4.2287 \text { E-05 }\end{array}$ & $3.4759 \mathrm{E}-06$ & $3.1943 \mathrm{E}-01$ & 17.0438 \\
$\begin{array}{l}\text { Intel 5-min returns } \\
-5.2863 \text { E-06 }\end{array}$ & $1.0241 \mathrm{E}-06$ & $-9.5237 \mathrm{E}-02$ & 21.2283 \\
$\begin{array}{l}\text { Intel 15-min returns } \\
-1.5839 \text { E-05 }\end{array}$ & $70.969 \mathrm{E}-07$ & $-1.7460 \mathrm{E}-01$ & 12.2681 \\
\hline
\end{tabular}

Moments for Cisco Systems and Intel stock returns are presented in this table. The time period goes from January 2, 1997 to December 31,1997; the data have been collected from Reuters. The number of observations for the period of analysis is $n=101,707$ and 10,171 for the Cisco systems series on 1-min and 10-min intervals and $n=20,352$ and 6784 for the Intel series on 5-min and 15-min intervals. 
The stochastic clock in formula (5) will precisely account for the representation of returns by different classes of distributions for different holding periods as already recommended by Fama (1965). From a modelling standpoint, it is interesting to observe that in some way, Mandelbrot and Clark agreed on their correction of normality of returns since a stable process is also a time-changed Brownian. For a stable process with index $\alpha$, where $\alpha$ is between 0 and 2, the Lévy measure is

$$
k(\mathrm{~d} x)=\frac{1}{x^{\alpha+1}} \mathrm{~d} x \text { for } x>0 .
$$

The characteristic function of a Brownian motion evaluated at an independent increasing stable process $T$ of index $\alpha$ is given by

$$
E[\exp (\mathrm{i} u W(T(t)))]=E\left[\exp \left(\frac{-u^{2} T(t)}{2}\right)\right]=\exp \left[-t\left(\frac{c}{2}\right)|u|^{2 \alpha}\right] .
$$

Hence the process $X(t)=W(T(t))$ is a stable process of index $2 \alpha$.

Another property that we can derive from Monroe's theorem relates to a finding which has been proposed in finance by several authors as an answer to the observed lepkokurtosicity of the return distribution as a mixture of normals.

Choosing for simplicity a discrete $f_{T}$ for $T$, we can write

$$
P(X(t) \in \mathrm{d} x)=\sum_{u} P(X(t) \in \mathrm{d} x / T(t)=u) f_{T}(u) .
$$

Now, assuming the independence of the processes $W$ and $T$, we obtain

$$
P(X(t) \in \mathrm{d} x)=\sum_{u} P(W(u) \in \mathrm{d} x) f_{T}(u) .
$$

Hence, the distribution of $Y$ appears as a mixture of normal distributions, where the mixing factor is the density of the time change, which itself accounts for the market activity measured by the volume or number of trades. It is interesting to note that in one of the most cited papers on the subject, Richardson and Smith (1994) have as a goal stated in the title the measure of daily flow information through the test of the mixture of distributions hypothesis.

Lastly, we can observe that the continuity of the process $(S(t))$ is equivalent to the continuity of the process $T(t)$. If $T(t)$ is continuous, then it may be written in the following form:

$$
T(t)=\int_{0}^{t} a(u) \mathrm{d} u+\int_{0}^{t} b(u) \mathrm{d} Z(u) .
$$

Because $T(t)$ is increasing, $b(u) \equiv 0$ and the time change is locally deterministic. This is an undesirable property since we believe $T(t)$ is related to locally random market activity like the arrival of orders or information; hence $T$ cannot be continuous. This is another argument in favor of the jump processes introduced in the following section. 


\section{Pure jump Lévy processes in finance}

The normal inverse Gaussian process, the hyperbolic motion, the variance gamma and the CGMY model are Lévy processes which share the property of being pure jump and infinite activity. Their empirical performance in fitting stock prices and equity option prices has been recently evidenced by a number of authors. In all four cases, the tractability of the characteristic function allows to recover option prices through the fast Fourier transform (see Carr and Madan, 1998). Hence, it suffices to assume the same structure of the process with possibly different parameters under the real probability measure $P$ and the risk-neutral probability measure $Q$ to test the goodness of fit both on stock and option data.

Generalized hyperbolic distributions were proposed by Barndorff-Nielsen (1977) for modelling the grain size of wind blown sand. They contain as subclasses the hyperbolic distribution and the normal inverse Gaussian model, respectively introduced in finance by Eberlein and Keller (1995) and Barndorff-Nielsen (1998).

\subsection{The normal inverse Gaussian model}

Its characteristic function is defined by three parameters:

$$
\phi_{\mathrm{NIG}}(u ; \alpha, \beta, t \delta)=\exp \left[-t \delta\left(\sqrt{\alpha^{2}-(\beta+\mathrm{i} u)^{2}}-\sqrt{\alpha^{2}-\beta^{2}}\right)\right] .
$$

The linearity of the log of the characteristic function with respect to time shows that it is an infinitely divisible-process with stationary independent increments.

We can also recognize the general property of being a time-changed Brownian motion is also satisfied by this Lévy process:

$$
X(t)=W(T(t))
$$

with the interesting feature that the time change $T$ may be chosen as an inverse Gaussian process independent of the directing Brownian motion $W$.

For $T$ positive, define $T_{t}^{v}$ the first time that a Brownian motion with drift $v$ reaches the positive level $t$. The density of $T_{t}^{v}$ is inverse Gaussian and its Laplace transform has a simple expression of the form

$$
E\left[\exp \left(-\lambda T_{t}^{v}\right)\right]=\exp \left[-t\left(\sqrt{2 \lambda+v^{2}}-v\right)\right] .
$$

If now we consider the Brownian motion with drift $\theta$ and volatility $\sigma$ computed at this Gaussian time, we define a new process $X_{\mathrm{NIG}}$ by

$$
X_{\mathrm{NIG}}(t ; \sigma, v, \theta)=\theta T_{t}^{v}+\sigma W\left(T_{t}^{v}\right) .
$$

This normal inverse Gaussian process has a characteristic function which is fairly simple: 


$$
\begin{aligned}
E\left[\mathrm{e}^{\mathrm{i} u X_{\mathrm{NIG}}(t)}\right] & =E\left[\exp \left(\mathrm{i} u \theta T_{t}^{v}-\frac{\sigma^{2} u^{2}}{2} T_{t}^{v}\right)\right] \\
& =E\left[\exp \left(\mathrm{i} u \theta-\frac{\sigma^{2} u^{2}}{2}\right) T_{t}^{v}\right] \\
& =\exp \left[-t \sqrt{v^{2}-2 \mathrm{i} u \theta+\sigma^{2} u^{2}}-v\right] \\
& =\exp \left(-t \sigma \sqrt{\frac{v^{2}}{\sigma^{2}}+\frac{\theta^{2}}{\sigma^{4}}-\left(\frac{\theta}{\sigma^{2}}+\mathrm{i} u\right)^{2}}-\frac{v}{\sigma}\right) .
\end{aligned}
$$

Denoting for simplication

$$
\left\{\begin{array}{l}
\beta=\frac{\theta}{\sigma^{2}} \\
\alpha^{2}=\frac{v^{2}}{\sigma^{2}}+\frac{\theta^{2}}{\sigma^{4}} \\
\delta=\sigma
\end{array}\right.
$$

we can write the NIG process as

$$
X_{\mathrm{NIG}}(T ; \alpha, \beta, \delta)=\beta \delta^{2} T_{t}^{\delta \sqrt{\alpha^{2}-\beta^{2}}}+\delta W\left(T_{t}^{\delta \sqrt{\alpha^{2}-\beta^{2}}}\right) .
$$

Conditioning on a jump of size $u$ in the time change, the move for the process $X$ is Gaussian with mean $\beta \delta^{2} u$ and variance $\delta^{2} u$. As seen earlier, the arrival rate of the jumps is given by the Lévy density, which in the case of the inverse Gaussian time $T$, has the following expression:

$$
k(u)=\frac{\exp \left[\frac{-\delta^{2}\left(\alpha^{2}-\beta^{2}\right)}{2} u\right]}{u^{3 / 2}} .
$$

It follows that the Lévy density for the NIG process is

$$
\begin{aligned}
& \int_{0}^{\infty} \frac{1}{\delta \sqrt{2 \pi u}} \exp \left(\frac{-\left(x-\beta \delta^{2} u\right)^{2}}{2 \delta^{2} u}\right) \frac{1}{u^{3 / 2}} \exp \left(\frac{-\delta^{2}\left(\alpha^{2}-\beta^{2}\right)}{2} u\right) \mathrm{d} u \\
& =\frac{1}{\delta} \int_{0}^{\infty} \frac{1}{\sqrt{2 \pi}} t^{-2} \exp \left(\frac{-\delta^{2} \alpha^{2}}{2} t-\frac{x^{2}}{2 \delta^{2} t}\right) \mathrm{d} t \\
& =\frac{\mathrm{e}^{\beta x}}{\delta} \int_{0}^{\infty} \frac{1}{\sqrt{2 \pi}} \exp \left(-s-\frac{x^{2} \alpha^{2}}{4 s}\right) s^{-2} \frac{\delta^{2} \alpha^{2}}{2} \mathrm{~d} s .
\end{aligned}
$$

We introduce the modified Bessel function $K_{\mathrm{a}}$ (see Abramovitz and Stegun, 1972):

$$
K_{a}(x)=\frac{1}{2}\left(\frac{x}{2}\right)^{a} \int_{0}^{\infty} \exp \left(-\left(t+\frac{x^{2}}{4 t}\right)\right) t^{-a-1} \mathrm{~d} t
$$

which allows to extract 


$$
\int_{0}^{\infty} \exp \left(-\left(t+\frac{x^{2}}{4 t}\right)\right) t^{-a-1} \mathrm{~d} t=2 K_{a}(x)\left(\frac{2}{x}\right)^{a} .
$$

Hence, the NIG Lévy density above written involves the Bessel function $K_{1}$ with index 1 and reduces to

$$
k_{\mathrm{NIG}}(X)=\sqrt{\frac{2}{\pi} \delta \alpha^{2}} \mathrm{e}^{\beta x} \frac{K_{1}(|x|)}{|x|} .
$$

We can observe that the integral of $k_{\mathrm{NIG}}$ over the real line is infinite; hence, the NIG process has infinite activity. Venter and de Jongh (2002) confirm the quality of the fitting obtained with the NIG process on a database of returns of the FT-Actuaries All-Share Index for the UK from January 1965 to December 1995. Morevover, they show that when VAR is the risk measure, the NIG based approach is found to be more robust than the extreme value theory (EVT) method for samples of sizes up to 250 and also in larger samples if the NIG distribution fits well. According to these authors, the EVT method should only be used in large samples if the NIG distribution does not fit adequately.

\subsection{The hyperbolic and generalized hyperbolic distributions}

The hyperbolic distribution has a density given by

$$
f_{(\alpha, \beta, \delta, \mu)}(x)=\frac{\sqrt{\alpha^{2}-\beta^{2}}}{2 \alpha \delta K_{1}\left(\delta \sqrt{\alpha^{2}-\beta^{2}}\right)} \exp \left(-\alpha \sqrt{\delta^{2}+(x-\mu)^{2}}+\beta(x-\mu)\right)
$$

where $K_{1}$ denotes the modified Bessel function with index 1 .

Barndorff-Nielsen and Halgreen (1977) show that the hyperbolic distribution can be represented as a mixture of normals, where the mixing distribution is a generalized inverse Gaussian with density

$$
h(x)=\frac{\sqrt{\omega / \gamma}}{2 K_{1}(\sqrt{\omega \gamma})} \exp \left\{-\frac{1}{2}\left(\gamma x^{-1}+\omega x\right)\right\} \text { for } x>0 .
$$

Let $\gamma=\delta^{2}$ and $\omega=\alpha^{2}-\beta^{2}$ and introduce a normal distribution with mean $\mu+\beta \sigma^{2}$ and variance $\sigma^{2}$ such that $\sigma^{2}$ is a random variable with distribution $h(x)$. Then the mixture is a hyperbolic distribution $f_{\alpha, \beta, \delta, \mu}$ defined above.

We observe that:

- the log-density is a hyperbola, hence the name (as opposed to a parabola for the log-density of the normal distribution),

- it provides heavier tails,

- it is characterized by four parameters: $\mu \in R, \delta>0$ and $0 \leqslant|\beta|<\alpha$,

- $\alpha$ and $\beta$ determine the shape ( $\beta$ being responsible for skewness),

- $\delta$ and $\mu$ are respectively scale and location parameters. 
The moment generating function of the hyperbolic motion is given by

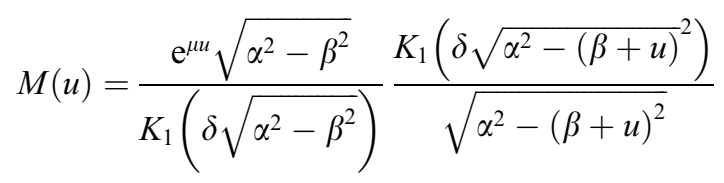

where $|\beta+u|<\alpha$.

Proof. It is just a straightforward computation of the integral

$$
M(u)=\int \mathrm{e}^{u x} f_{(\alpha, \beta, \delta, \mu)}(x) \mathrm{d} x .
$$

Hence all moments of the hyperbolic distribution exist and can be obtained through the derivatives of $M$ evaluated at $t=0$.

The Lévy-Khintchine representation of the characteristic function in the symmetric centered case $(\beta=\mu=0)$ has the following expression:

$$
\phi(u)=\exp \int\left(\mathrm{e}^{\mathrm{i} u x}-1-\mathrm{i} u x\right) g(x) \mathrm{d} x
$$

with

$$
g(x)=\frac{1}{|x|}\left[\int_{0}^{\infty} \frac{\mathrm{e}^{-\sqrt{2 y+\alpha^{2}|x|}} \mathrm{d} y}{\pi^{2} y\left(J_{1}^{2}(\delta \sqrt{2 y})+Y_{1}^{2}(\delta \sqrt{2 y})\right)}+\mathrm{e}^{-\alpha|x|}\right]
$$

where $J_{1}$ and $Y_{1}$ are Bessel functions.

Using the asymptotics of the various Bessel functions, one can deduce that $g(x) \sim 1 / x^{2}$ for $x \rightarrow 0$; hence every path of the process has infinitely many jumps in any finite interval. However, the magnitude of the jumps is such that the moment generating function exists, which is in contrast to the $\alpha$-stable Lévy process.

The generalized hyperbolic distribution involves an extra-parameter $\lambda$ and has the following density:

$$
\begin{aligned}
f_{\mathrm{GH}}(x ; \lambda, \alpha, \beta, \delta, \mu)= & a(\lambda, \alpha, \beta, \delta)\left(\delta^{2}+(x-\mu)^{2}\right)^{\frac{\left(\lambda-\frac{1}{2}\right)}{2}} \\
& \times K_{\lambda-\frac{1}{2}}\left(\alpha \sqrt{\delta^{2}+(x-\mu)^{2}} \exp (\beta(x-\mu))\right)
\end{aligned}
$$

where

$$
a(\lambda, \alpha, \beta, \delta)=\frac{\left(\alpha^{2}-\beta^{2}\right)^{\lambda / 2}}{\sqrt{2 \pi} \alpha^{\lambda-\frac{1}{2}} \delta^{\lambda} K_{\lambda}\left(\delta \sqrt{\alpha^{2}-\beta^{2}}\right)}
$$

is the normalizing constant. $K_{v}$ denotes as before the Bessel function with index $v$. The extra-parameter $\lambda$ characterizes certain sub-classes and has essentially an impact on the heaviness of the tails. For $\lambda=1$, we recover the sub-class of hyperbolic dis- 


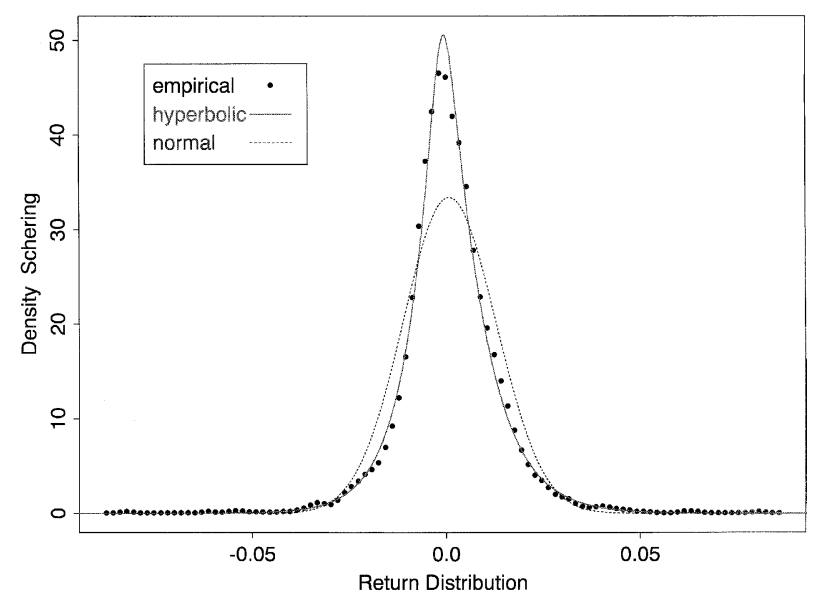

Fig. 1. Repeal Schering stock distribution fitted by the hyperbolic model.

tributions, for $\lambda=-1 / 2$ the normal inverse Gaussian. The fact that generalized hyperbolic distributions are infinitely divisible allows to generate a Lévy process $\left(X_{t}\right)$ such that the distribution of $X_{1}$ has the density $f_{\mathrm{GH}}$ defined earlier. This process is defined by Eberlein (1999) as the generalized hyperbolic Lévy motion.

Eberlein et al. (1998) show that hyperbolic distributions allow an almost perfect fit to financial data, both in spot and derivative markets; their empirical analysis investigated in particular major German stocks such as Deutsche Bank, Thyssen, the DAX index as well as options on these various underlyings. Fig. 1 shows the distribution of returns for the Schering stock, a pharmaceutical company included in the DAX index.

\subsection{The variance gamma model}

Madan et al. (1998) introduce the process defined by an arithmetic Brownian motion with drift $\theta$ and volatility $\sigma$, time-changed by an increasing Gamma process with unit mean and variance $v$, resulting in the three parameter process

$$
X(t ; \sigma, v, \theta)=\theta G(t ; v)+\sigma W(G(t ; v))
$$

where $G(t ; v)$ is the Gamma process and $W(t)$ is a standard Brownian motion. The probability density of the gamma process with mean rate $t$ and variance $v t$ is well known:

$$
f(u)=\frac{u^{\frac{t}{v}-1} \mathrm{e}^{\frac{-u}{v}}}{v^{\frac{t}{v}} \Gamma\left(\frac{t}{v}\right)}
$$

where $\Gamma(x)$ denotes the classical gamma function. Its Laplace transform reduces to

$$
E\left[\exp \left(-\lambda G_{t}^{v}\right)\right]=(1+\lambda v)^{-t / v} .
$$

It results that the variance gamma process has a particularly simple characteristic function, 


$$
\phi_{v G}(u)=\left(\frac{1}{1-\mathrm{i} \theta v u+\frac{\sigma^{2} v}{2} u^{2}}\right)^{t / v},
$$

obtained by conditioning on the time change and using (7) for

$$
\lambda=\frac{\sigma^{2} u^{2}}{2}-\mathrm{i} \theta u \text {. }
$$

The moment equations can be uniquely solved for the parameters:

$$
\begin{aligned}
& \text { variance }=\theta^{2} v+\sigma^{2}, \\
& \text { central third moment }=2 \theta^{3} v^{2}+3 \sigma^{2} \theta v, \\
& \text { central fourth moment }=3 \text { variance }^{2}+3 \sigma^{4} v+12 \sigma=+\theta^{2} v^{2}+6 \theta^{4} v^{3} .
\end{aligned}
$$

Geman et al. (2001) show that the variance gamma process may be expressed as the differences of two independent gamma processes.

$$
X(t)=G_{\mathrm{p}}(t)-G_{\mathrm{n}}(t)
$$

where $G_{\mathrm{p}}(t)$ may be interpreted as the price change resulting from "positive" shocks and $G_{\mathrm{n}}(t)$ the price change resulting from "negative" shocks. It suffices for instance to write

$$
\frac{1}{1-\mathrm{i} \theta v u+\sigma^{2} v \frac{u^{2}}{2}}=\left(\frac{1}{1-\mathrm{i} u \eta_{\mathrm{p}}}\right)\left(\frac{1}{1+\mathrm{i} u \eta_{\mathrm{n}}}\right)
$$

where

$$
\begin{aligned}
& \eta_{\mathrm{p}}-\eta_{\mathrm{n}}=\theta v \\
& \eta_{\mathrm{p}} \eta_{\mathrm{n}}=\frac{\sigma^{2} v}{2}
\end{aligned}
$$

which in turn results into

$$
\begin{aligned}
& \eta_{\mathrm{p}}=\left(\sqrt{\frac{\theta^{2} v^{2}}{4}+\frac{\sigma^{2} v}{2}}-\frac{\theta v}{2}\right)^{-1}, \\
& \eta_{\mathrm{n}}=\left(\sqrt{\frac{\theta^{2} v^{2}}{4}+\frac{\sigma^{2} v}{2}}+\frac{\theta v}{2}\right)^{-1} .
\end{aligned}
$$

The fact that the VG process $X$ may be written as the difference of the processes $G_{\mathrm{p}}(k)$ and $G_{\mathrm{n}}(k)$ implies that it is a finite variation process. Moreover, the knowledge of the positive and negative moves allows the determination of the Lévy density under the following form: 


$$
k_{\mathrm{VG}}(x)= \begin{cases}C \frac{\exp (-M x)}{x}, & x>0, \\ C \frac{\exp (-G|x|)}{|x|}, & x<0,\end{cases}
$$

where

$$
C=\frac{1}{v} ; G=\frac{1}{\eta_{\mathrm{n}}} ; M=\frac{1}{\eta_{\mathrm{p}}} .
$$

Appearing as a scale factor in the Lévy measure both for positive and negative moves, the constant $C$ characterizes the general activity intensity of the process while the parameters $G$ and $M$ define the speed at which arrival rates decline with the size of the move. In a parallel interpretation, we may come back to the moment equations written earlier and observe that the parameter $\theta$ provides skewness to the distribution as it enhances the left tail when negative by both decreasing $G$ and simultaneously increasing $M$; the opposite holds for $\theta>0$. The parameter $v$ provides kurtosis which, in the absence of skew $(\theta=0)$, is equal to $3(1+v)$.

In the spirit of Section 3 , it is interesting to note that $v$ is non-zero whenever the time change $G(t, v)$ is stochastic, which equivalently corresponds to the situation of excess kurtosis created by stochastic volatility. Lastly, we can observe that the integral of $k$ on the real line, which is the situation that we described as infinite activity for the Lévy measure.

\subsection{The CGMY process}

As seen in the previous paragraphs, the NIG process has infinite activity and infinite variation; the VG process has finite variation and infinite activity. To represent these various possibilities for different values of the parameter set, Carr et al. (2002) introduce the following Lévy density:

$$
k_{\mathrm{CGMY}}(x) \begin{cases}\frac{C \mathrm{e}^{-M x}}{x^{1+Y}}, & x>0, \\ \frac{C \mathrm{e}^{-G|x|}}{|x|^{1+Y}}, & x<0 .\end{cases}
$$

The parameter $Y$ captures the "fine" structure of the process in the following way: for low values of $Y$, the Lévy density integrates to a finite value yielding a process of finite activity. At the same time, the integral of $|x|$ times the Lévy density is also finite and the process has finite variation, as a compound Poisson process. For higher values of $Y$ (typically between 0 and 1), the process has infinite activity and infinite variation, like the VG process. For yet higher values of $Y$ (between 1 and 2), the process has infinite activity and infinite variation like the NIG process.

The CGMY characteristic function is obtained by integration as

$$
\log \left[\phi_{\mathrm{CGMY}}(u)\right]=C t \Gamma(-y)\left\{(M-\mathrm{i} u)^{Y}-M^{Y}+(G+\mathrm{i} u)^{Y}-G^{Y}\right\} .
$$

In order to envision the possibility of moves other than jumps, the CGMY process is extended to CGMYe model by addition of a diffusion component 


$$
X_{\mathrm{CGMYe}}(t)=X_{\mathrm{CGMY}}(t)+\eta W(t)
$$

where $\eta$ is a real number.

The CGMYe characteristic function is given by

$$
\log \left[\phi_{\mathrm{CGMYe}}(u)\right]=\log \left[\phi_{\mathrm{CGMY}}(u)\right]-\eta^{2} u^{2} \frac{t}{2} .
$$

The CGMYe stock price process is defined by

$$
S(t)=S(0) \exp \left\{\left(\mu+\omega-\frac{\eta^{2}}{2}\right) t+X_{\mathrm{CGMYe}}(t)\right\}
$$

where

$$
\omega=\frac{-1}{t} \log \left[\phi_{\mathrm{CGMY}}(I)\right]
$$

ensures that the mean rate of return is $\mu$.

The log characteristic function of the log stock price is

$$
\log \left[\phi_{\ln s}(u)\right]=\mathrm{i} u\left[(\ln S(0))+\left(\mu+\omega-\frac{\eta^{2}}{2}\right) t\right]+\log \left[\phi_{\mathrm{CGMYe}}(u)\right] .
$$

The higher moments of the CGMYe process are

$$
\begin{aligned}
& E[X-E(X)]^{2}=\eta^{2}+\int_{-\infty}^{+\infty} x^{2} k(x) \mathrm{d} x, \\
& E[X-E(X)]^{3}=\int_{-\infty}^{+\infty} x^{3} k(x) \mathrm{d} x, \\
& E[X-E(X)]^{4}=3 \text { (variance) }+\int_{-\infty}^{+\infty} x^{4} k(x) \mathrm{d} x .
\end{aligned}
$$

Carr et al. (2002) report on the goodness of fit obtained with the CGMY model on US individual stocks and equity indices. Equity indices such as the SPX behave like processes of infinite activity and finite variation. Quite interestingly, testing the CGMYe against the CGMY representation shows the absence of a diffusion component in equity indices, hence the conjecture that diffusion components possibly existing in individual stock prices are diversified away in the index whose moves are pure jumps. This is a message quite different from the assumption of zero market price of jump risk in jump-diffusion models such as Merton (1976); it is in agreement with the perception that on any day the whole market moves together, sometimes because of a sharp price change in a single name, as observed over the recent years. Fig. 2 shows the excellent fit obtained on a database of the SPX index over the period January 1, 1994 to December 31, 1998 using the pure jump CGMY without any diffusion term.

The same mathematical structure may be used for the risk-neutral process where the mean is set to equal the interest rate and the three other parameters are determined by matching option prices. More precisely, the stock price process is modelled under the risk-adjusted probability measure $Q$ by 


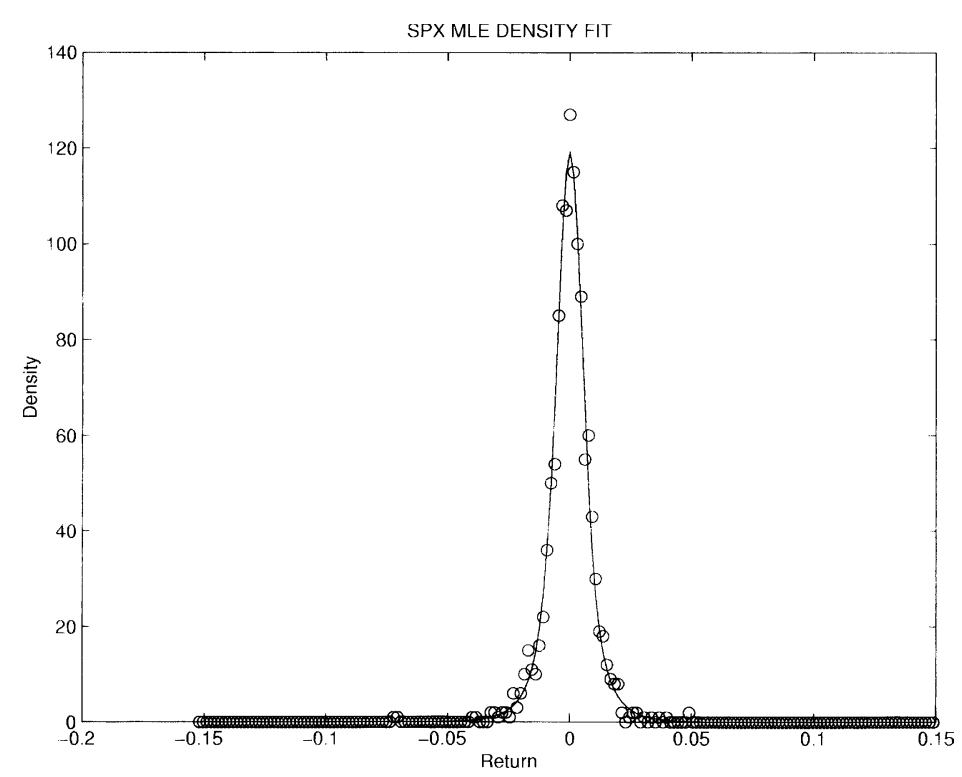

Fig. 2. SPX index distribution fitted by the CGMY model.

$$
S(t)=S(0) \exp \left[(r-q+\omega) t+X_{C^{\prime} \mathbf{G}^{\prime} \mathbf{M}^{\prime} \mathbf{Y}^{\prime}}(t)\right]
$$

where $\omega=-(1 / t) \ln \left[\phi_{\mathrm{C}^{\prime} \mathrm{G}^{\prime} \mathrm{M}^{\prime} \mathrm{Y}^{\prime}}(-i)\right]$ ensures that the mean rate of return is $(r-q)$, denoting the dividend yield.

The Fourier transform of a standard European call price may be expressed in terms of the log of the strike as

$$
\gamma(u)=\int_{-\infty}^{+\infty} \mathrm{e}^{\mathrm{i} u k} \mathrm{e}^{\alpha k} C(k) \mathrm{d} k
$$

where the call has a strike $\mathrm{e}^{k}$ and the term $\mathrm{e}^{\alpha k}$ is meant to ensure the convergence of the integral.

Call prices are then recovered by inversion,

$$
C(k)=\left(\mathrm{e}^{-\alpha k} / 2 \pi\right) \int_{-\infty}^{+\infty} \mathrm{e}^{\mathrm{i} u k} \gamma(u) \mathrm{d} u,
$$

and the parameters under the risk-neutral measure are derived by calibration to option market prices.

Figs. 3-5 show the quality of the fitting obtained with a pure jump CGMY (properly risk-neutralized) in the case of the Stoxx $50 \mathrm{E}$ volatility surface. The calibration is superior when adjusting separately short maturities on one hand and long maturities on the other hand. A more complex version of the CGMY model, currently under completion, will allow to handle all strikes and maturities at once. 


\section{Stoxx 50 E implied volatility surface}

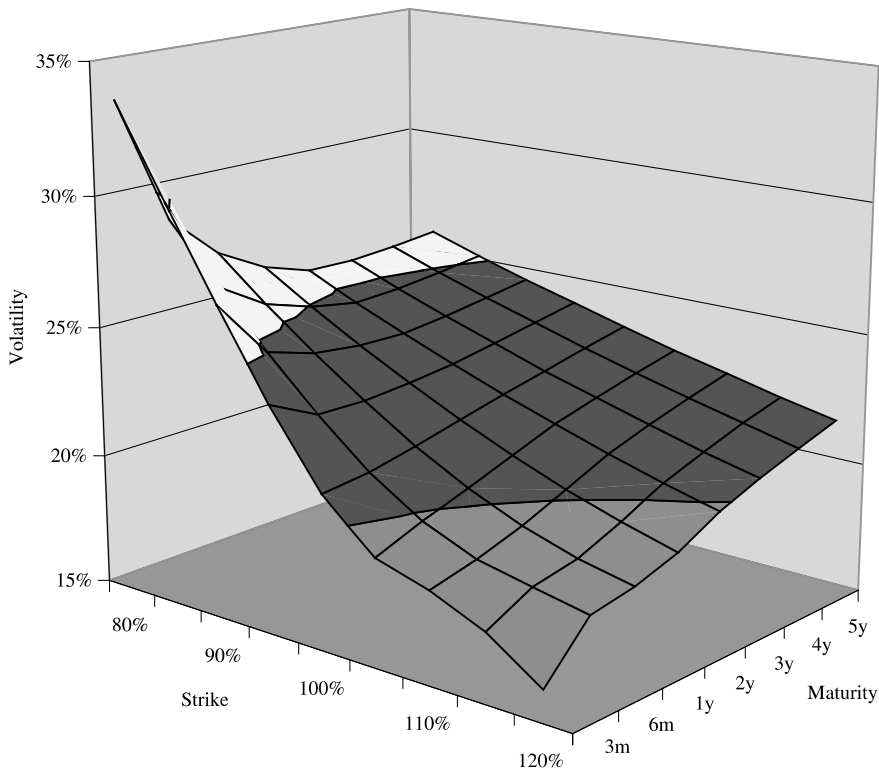

Fig. 3.

CGMY (calibrated to short maturity) implied volatility surface

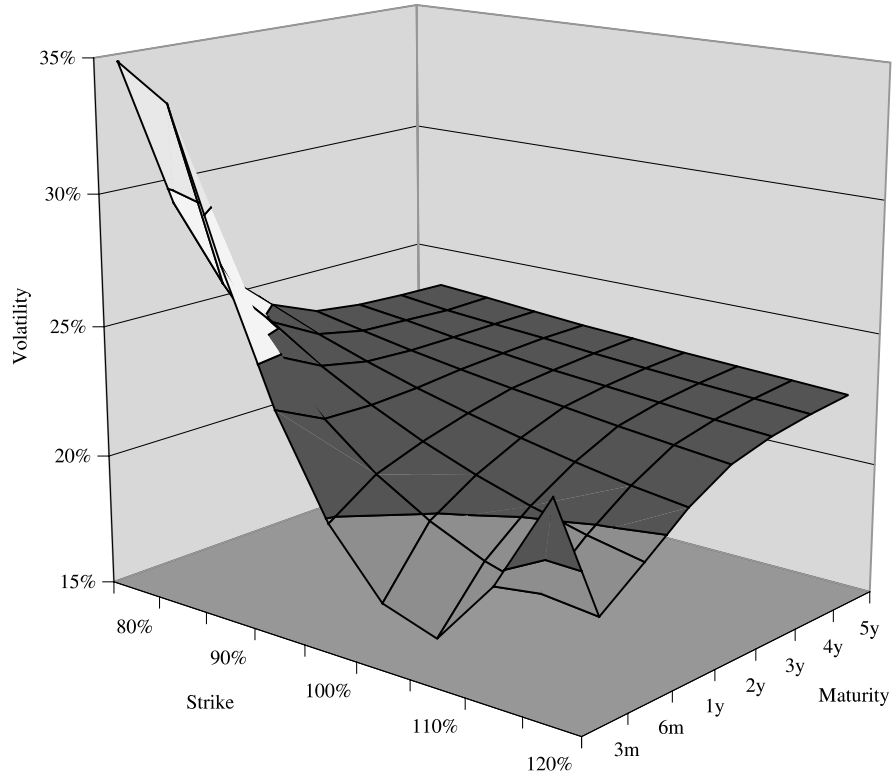

Fig. 4. 
CGMY (calibrated to long maturity) implied volatility surface

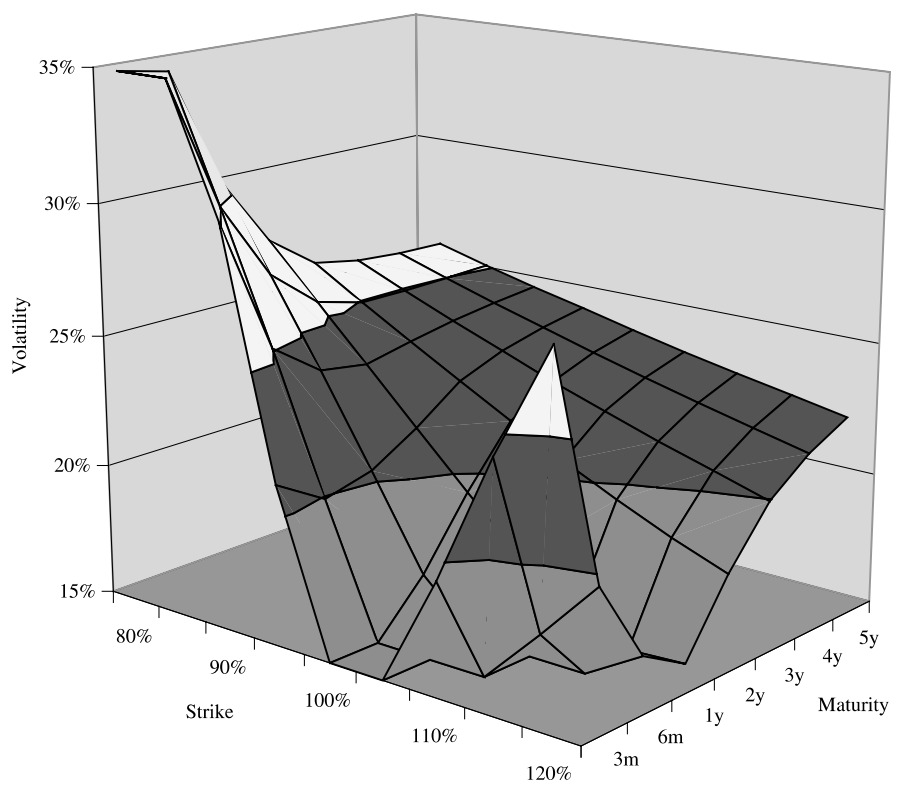

Fig. 5 .

\section{Conclusion}

We argue in this paper that pure jump Lévy processes with finite variation and infinite activity are better representations of stock price dynamics than the classical diffusions or jump-diffusion models. Their representation as time-changed Brownian motion allows to shift the focus of attention to modelling the time change which itself reflects the intensity of the economic activity through news arrival and trades. We provide examples which have lately been evidenced to perform very well in fitting financial data in US and European equity markets. The existence of explicit expressions for the characteristic functions of the NIG/hyperbolic motion and CGMY processes reduces the complexity of the underlying Lévy measures to a simple representation, quite amenable for statistical estimation.

\section{References}

Abramovitz, M., Stegun, I., 1972. Handbook of Mathematical Functions. Applied Mathematics Series, vol. 55. Springer, Berlin.

Ané, T., Geman, H., 1999. Stochastic volatility and transaction time: An activity-based volatility estimator. The Journal of Risk 2, 57-69.

Ané, T., Geman, H., 2000. Order flow, transaction clock and normality of asset returns. Journal of Finance. 
Barndorff-Nielsen, O., Halgreen, O., 1977. Infinite divisibility of the hyperbolic and generalized inverse Gaussian distributions. Zeitschrift für Wahrscheinlichkeitstheorie und Verwandte Gebiete 38, 309-312.

Barndorff-Nielsen, O., 1998. Processes of normal inverse Gaussian type. Finance and Stochastics 2, 41-68.

Black, F., Scholes, M., 1973. The pricing of option and corporate Liabilities. Journal of Political Economy 81, 637-654.

Carr, P., Geman, H., Madan, D., Yor, M., 2002. The fine structure of asset returns: An empirical investigation. Journal of Business.

Carr, P., Madan, D., 1998. Option valuation using the fast Fourier transform. Journal of Computational Finance 2, 61-73.

Clark, P., 1973. A subordinated stochastic process with finite variance for speculative prices. Econometrica 41, 135-155.

Eberlein, E., Keller, U., 1995. Hyperbolic distributions in finance. Bernoulli 1, 281-299.

Eberlein, E., Keller, U., Prause, K., 1998. New insights into smile, mispricing and value at risk: The hyperbolic model. Journal of Business 71 (3).

Eberlein, E., 1999. Application of generalized hyperbolic Lévy motions to finance. Lévy Processes. Birkhaüser, Basel.

Fama, E., 1965. The behavior of stock market prices. Journal of Business 38, 34-105.

Geman, H., Ané, T., 1996. Stochastic Subordination, RISK, September.

Geman, H., Madan, D., Yor, M., 2001. Times changes for Lévy processes. Mathematical Finance 11, 7996.

Geman, H., Yor, M., 1993. Bessel processes, Asian options and perpetuities. Mathematical Finance 2, $349-375$.

Hull, J.C., White, A.D., 1987. The pricing of options on assets with stochastic volatilities. Journal of Finance 42 (2), 281-300.

Jones, C., Kaul, G., Lipson, M., 1994. Transactions, volume and volatility. Review of Financial Studies 7, 631-651.

Karpoff, J., 1987. The relation between price changes and trading volume: A survey. Journal of Financial and Quantitative Analysis 22, 109-126.

Madan, D., Carr, P., Chang, E., 1998. The variance gamma process and option pricing model. European Finance Review 2, 79-105.

Mandelbrot, B., 1963. New methods in statistical economics. Journal of Political Economy 61, 421-440.

Merton, R.C., 1973. Theory of rational option pricing. Bell Journal of Economics and Management Science 4, 141-183.

Merton, R.C., 1976. Option pricing when underlying stocks are discontinuous. Journal of Financial Economics 3, 125-144.

Monroe, I., 1978. Processes that can be embedded in Brownian motion. The Annals of Probability 6 (1), $42-56$

Richardson, M., Smith, T., 1994. A direct test in the mixture of distributions hypothesis: Measuring the daily flow of information. Journal of Financial and Quantitative Analysis 29, 101-116.

Venter, J., de Jongh, P., 2002. Risk estimation using the normal inverse Gaussian distribution. The Journal of Risk 2, 1-25. 